\title{
Whole-body hybrid PET/MRI: ready for clinical use?
}

\author{
Osman Ratib • Thomas Beyer
}

Published online: 5 April 2011

(C) Springer-Verlag 2011

Since its introduction in 1998 [1], combined PET/CT has gained clinical acceptance as an oncology imaging modality providing superior accuracy in tumour staging and assessment of oncology therapy response compared to PET or $\mathrm{CT}$ alone $[2,3]$ and to both imaging modalities viewed side-by-side [4, 5]. The PET technology available in combined PET/CT has advanced significantly over the years and now supports emission imaging of the torso from head to mid-thigh with isotropic sub-centimetre resolution in $10 \mathrm{~min}$, or less. The integration of high-end multidetector CT in dual modality tomographs marks a trend towards performing contrast-enhanced diagnostic CT studies, which has progressively increased the clinical relevance of integrated PET/CT imaging protocols. Undisputedly, these changes in diagnostic pathways require the close collaboration and even cross-specialist training of nuclear medicine specialists and radiologists.

With the concurrent evolution of MR imaging technologies and sequences, MR studies can provide a wealth of diagnostic information regarding soft-tissue analysis, tumour detection, tissue characterization and functional imaging. It is not uncommon today for patients to undergo an MR study in addition to a PET/CT study as part of their clinical workup for detection and staging of cancers if soft-

O. Ratib ( $\square)$

Department of Medical Imaging and Information Sciences,

University Hospital of Geneva,

24, rue Micheli-du-Crest,

1205 Geneva, Switzerland

e-mail: osman.ratib@hcuge.ch

T. Beyer

cmi-experts $\mathrm{GmbH}$,

Zurich, Switzerland tissue characterization and morphological information that $\mathrm{CT}$ alone cannot provide are required. It is, therefore, quite rational to anticipate that combined PET/MR examinations will potentially become more valuable than PET/CT imaging followed by a complementary MR examination [6] in PET indications where MR outperforms CT. It has even been suggested that whole-body MR imaging (WBMRI) could replace PET/CT [7]. However, such these types of enthusiastic reports on the excellent diagnostic performance of stand-alone MR imaging are based mostly on using diffusion weighted (DW) sequences that show particularly high sensitivity at the cost of low specificity, and, therefore, may be of limited value in a cost-conscious health-care system.

\section{Technical innovation in combined PET/MR}

The idea of combining PET and MR arose around the same time that PET/CT was conceptualized. In the early 1990s Simon Cherry and Paul Marsden saw a need for PET/MR in small-animal imaging studies to combine high soft-tissue contrast with molecular information provided by PET $[8,9]$. PET/MR was destined to remain in the preclinical arena for another decade until, in 2006, the first simultaneous MR and PET images of the human brain were acquired [10]. A major challenge for bringing PET and MR technologies physically together is the interferences between the high-field magnets and the electronics of the PET scintillation detectors [11]. One solution for PET detectors to be compatible with MR, is to replace photomultipliers with avalanche photodiodes (APD) that are less sensitive to magnetic fields, or to use alternative solid-state technologies, which have been proposed in prototype animal PET systems [12]. It is 
worth noting that, unlike combined PET/CT, dual modality PET/MR started out in the preclinical arena before being adopted clinically, much supported by industry. Today three concepts for human PET/MR systems are vividly and often controversially discussed: separate PET, or PET/CT and MR gantries operated in different rooms (A), PET and MR gantries arranged in the direction of the main scanner axis with a patient handling system mounted in between (B), and a fully integrated PET/MR system with simultaneous acquisition (C).

The integrated design (C), first presented in 2006, and also the most challenging, is based on a PET detector ring designed as an insert that can be placed inside a 3-T MR scanner (Siemens Healthcare). This prototype system (BrainPET) was anticipated for brain imaging only [10]. The PET insert has an internal diameter of $35.5 \mathrm{~cm}$ and comprises LSO (lutetium oxyorthosilicate) detector blocks made of $2.5-\mathrm{mm}$ crystals coupled to an array of APDs that have been shown to be operational in magnetic fields of up to $7 \mathrm{~T}$ [8]. Four such units are now in research operation at the University of Tübingen, Forschungszentrum Jülich, MGH Boston and Emory University Atlanta.

The coplanar PET/MR concept (B), first presented in 2010 , is based on a tandem design of a whole-body time-offlight (TOF) PET system and a 3-T MR system (Philips Healthcare) with a rotating table platform in between. Through minor modifications of the PET detector system (e.g. orientation of the photomultiplier tube, minor shielding) the PET gantry can be operated in close proximity to the 3-T MR system. Two such whole-body units have been installed and tested in clinical routine in Mount Sinai Hospital in New York and at the University Hospital of Geneva, and have been certified since January 2011 for routine clinical use.

Design (A) was proposed by GE Healthcare in late 2010. It is available only as prototype technology and has been installed and tested at the University Hospital of Zurich. This design is based on a combination of a dual modality PET/CT and a 3-T MR system that are operated side-by-side in separate adjacent rooms; patients are shuttled from one system to the other without getting off the bed.

In an extension to the integrated design concept (C), a similar system was proposed in late 2010 that merges a whole-body PET with a 3-T MR system to allow simultaneous torso and whole-body imaging. Just like the BrainPET PET/MR prototype, this system is based on LSO-APD PET detector technology that is integrated into the MR gradient coil system offering a $60-\mathrm{cm}$ gantry opening (versus a $35-\mathrm{cm}$ gantry opening for the brain prototype). Since December 2010 two prototypes have been installed in clinical settings at the Technical University of Munich and the University of Tübingen.

\section{PET/MR or PET/CT?}

Since the introduction of PET/CT into clinical practice in 1998, clinical evidence for the diagnostic superiority of PET/CT over CT and PET acquired separately has been growing. This pertains to a number of indications, primarily in oncology [13-15]. It is conceivable that similar advantages over stand-alone imaging could be expected from combining PET with high-resolution MR images. However, the acquisition of whole-body MR data with adequate anatomical resolution and within a reasonable total examination time, matching that of a whole-body PET/CT study remains a challenge. As such, the existence of PET/CT may be the biggest challenge to the concept of PET/MR, both in terms of the diagnostic accuracy and logistical benefits.

Nonetheless, in several instances the information provided by MR cannot be obtained by CT [16]. For example, MR is advantageous in soft-tissue characterization, such as in parenchymal organs, the brain and bone lesions. Therefore, the concept of combining PET and MR continues to be of interest and clinical attention will increase in particular as a combination of whole-body MR techniques with specific tracer imaging in PET may provide incremental diagnostic accuracy. In addition, further clinically relevant information can be gathered by MR within the same examination procedure. For example, advanced MR imaging techniques can provide functional information, for example MR spectroscopy (MRS), diffusionweighted and perfusion imaging, and functional MR imaging (fMRI). As an example, MR breast imaging has a high sensitivity in the detection of breast lesions but a relatively low specificity [17]. In comparison, $\left[{ }^{18} \mathrm{~F}\right] \mathrm{FDG}$ $\mathrm{PET} / \mathrm{CT}$ imaging has gained a significant role in the detection and staging of breast cancer patients with a higher specificity than MR mammography $[18,19]$. Fusion of MR and PET data should, therefore, combine the high sensitivity of MR imaging for detection of breast lesions and the high specificity of PET for differentiating a benign from a malignant lesion [6]. Other domains for combined PET/MR are likely to be prostate cancer and head/neck cancer imaging. Finally, replacing the CT in PET/CT with MR could significantly reduce the effective patient exposure, although this may be less relevant in oncology and in elderly patients given their reduced life expectancy. It is, however, beneficial in younger patients with potentially curable disease and in patients with non-oncological indications.

Despite the promising potential of dual modality PET/ MR, a number of methodological and clinical concerns await resolution before PET/MR can become more widely adopted in clinical routine. First, this relates to the derivation of attenuation correction (AC) factors from PET/MR data. Several techniques for calculation MR AC 
based on MR image segmentation of different tissue characteristics of the body have been implemented and tested [20], but numerous technical challenges for accurate AC remain, including compensation for MR image truncation and correction for RF coils and accessories. Second, PET/MR imaging protocols have a direct impact on patient throughput. Adding emission acquisition time and multisequence clinical MR imaging time could result in total examination times that are significantly longer than those of a comparable PET/CT study. Therefore, the combination of PET and $\mathrm{MR}$ in a single imaging modality requires modified imaging protocols that yield competitive examination times. Otherwise, two already long examination times will add up to an examination time not acceptable in clinical routine. While advanced MR imaging sequences can provide relevant diagnostic information, they cannot be applied to whole-body imaging. Specific imaging sequences may have to be restricted to organs and body sections that benefit most from such imaging protocols, such as body regions with an abnormal metabolic pattern as seen on PET. Conversely, with higher efficiency PET systems using TOF technology, imaging time can be reduced essentially by half while maintaining equivalent or better image quality than non-TOF PET systems. This should allow shorter PET imaging times that leave more flexibility for additional complementary MR sequences to be performed. Recently it has been suggested that TOF can also be helpful in reducing the effects of inconsistent data, such as erroneous normalization, poor scatter estimates and mismatched or inaccurate attenuation maps [21].

Most clinical protocols today require between two and five additional MR series with dedicated protocols on specific organs or body sections. These additional sequences can also be completed in under 30 minutes, allowing a diagnostic PET/MR scan to be completed within an hour. While such a study is longer than a standard diagnostic PET/CT study, it is still shorter than the total time that a PET/CT and an MRI study would take today if performed on two separate systems at different times. This clearly benefits patients who need both PET/CT and MR studies in their clinical workup. We also believe that using the results of the PET study to identify areas where additional MR sequences must be applied could significantly reduce the time for a diagnostic MR scan by limiting the number and extent of these additional MR sequences by applying them only to limited areas where suspicious PET uptake is identified. Other challenges for the routine use of PET/MR include methodological aspects, such as the fact that CT is the modality of choice for the characterization of lung lesions. In some patients with metal implants and pacemakers, MR imaging may not be employed at all. Also, the number of patients unable to undergo the study due to claustrophobia is higher with MR than with CT.
Overall, we believe that in oncology applications MR images can bring additional relevant diagnostic information to PET images and the combination of the two modalities can offer more accurate and specific diagnostic performance than PET/CT alone [6]. Coregistered anatomy and metabolic images should enable better staging and more accurate lesion identification in a variety of tumour entities such as liver and bone metastases, brain tumours, and prostate, gynaecological, breast and head and neck cancers. In thoracic tumours, however, PET/CT will still be preferred. However, the added diagnostic value provided by MR over CT and PET must be proven to be valuable and valuable in the context of a presumably longer imaging time if PET/MR is performed instead of PET/CT.

In follow-up studies of patients undergoing specific oncological treatment, PET/MRI could become the modality of choice offering a more accurate and quantitative assessment of patient tumour response to treatment. Special protocols focused on specific body regions could use much shorter MR sequences than those used for diagnostic purposes. Repeated PET/MRI studies as part of patient treatment monitoring would have the major advantage of much lower radiation exposure than conventional PET/CT used in most cases today. With the emergence of new tracers, it is also expected that more specific and accurate PET protocols will emerge for diagnosis and follow-up of cancer, and also for prediction and assessment of patient response to treatments [22]. The combination of PET and MR images may be ideal for anatomical and metabolic assessment of protocols and clinical pathways for oncology [8].

While hybrid PET/MR may certainly not replace PET/ $\mathrm{CT}$ in all cases, it has the potential to improve workflow and diagnostic accuracy in patients who already require both a PET/CT and an MR examination as part of their clinical workup.

\section{References}

1. Townsend DW, Beyer T, Kinahan PE, Meltzer CC, Brun T, Nutt R, et al. The SMART Scanner: A combined PET/CT tomograph for clinical oncology. Radiology. 1998;209P:169-70.

2. Collins CD. PET/CT in oncology: for which tumours is it the reference standard? Cancer Imaging. 2007;7 Spec No A:S77-87.

3. Lonsdale MN, Beyer T. Dual-modality PET/CT instrumentation today and tomorrow. Eur J Radiol. 2010;73:452-60.

4. Antoch G, Saoudi N, Kuehl H, Dahmen G, Mueller SP, Beyer $\mathrm{T}$, et al. Accuracy of whole-body dual-modality fluorine-18-2fluoro-2-deoxy-D-glucose positron emission tomography and computed tomography (FDG-PET/CT) for tumor staging in solid tumors: comparison with CT and PET. J Clin Oncol. 2004;22:4357-68.

5. Bar-Shalom R, Yefremov N, Guralnik L, Gaitini D, Frenkel A, Kuten A, et al. Clinical performance of PET/CT in evaluation of 
cancer: additional value for diagnostic imaging and patient management. J Nucl Med. 2003;44:1200-9.

6. Antoch G, Bockisch A. Combined PET/MRI: a new dimension in whole-body oncology imaging? Eur J Nucl Med Mol Imaging. 2009;36 Suppl 1:S113-20.

7. Liang X, Congxin W, Yi Z, Jinwen H, Chuanfu L. Whole-body diffusion-weighted MR imaging with fat suppression by using STIR: Clinical utility for detection of primary malignancies in patients initially presented with metastasis. Eur J Radiol. 2010. Epub 2010 Mar 22. doi:10.1016/j.ejrad.2010.02.013

8. Pichler BJ, Kolb A, Nagele T, Schlemmer HP. PET/MRI: paving the way for the next generation of clinical multimodality imaging applications. J Nucl Med. 2009;51:333-6.

9. Shao Y, Cherry SR, Farahani K, Meadors K, Siegel S, Silverman RW, et al. Simultaneous PET and MR imaging. Phys Med Biol. 1997;42:1965-70.

10. Schlemmer HP, Pichler BJ, Schmand M, Burbar Z, Michel C, Ladebeck R, et al. Simultaneous MR/PET imaging of the human brain: feasibility study. Radiology. 2008;248:1028-35.

11. Slates RB, Farahani K, Shao Y, Marsden PK, Taylor J, Summers $\mathrm{PE}$, et al. A study of artefacts in simultaneous PET and MR imaging using a prototype MR compatible PET scanner. Phys Med Biol. 1999;44:2015-27.

12. Wehrl HF, Judenhofer MS, Wiehr S, Pichler BJ. Pre-clinical PET/MR: technological advances and new perspectives in biomedical research. Eur J Nucl Med Mol Imaging. 2009;36 Suppl 1:S56-68.

13. Fletcher JW, Kymes SM, Gould M, Alazraki N, Coleman RE, Lowe VJ, et al. A comparison of the diagnostic accuracy of $18 \mathrm{~F}$ FDG PET and CT in the characterization of solitary pulmonary nodules. J Nucl Med. 2008;49:179-85.
14. Yang W, Fu Z, Yu J, Yuan S, Zhang B, Li D, et al. Value of PET/ CT versus enhanced CT for locoregional lymph nodes in nonsmall cell lung cancer. Lung Cancer. 2008;61:35-43.

15. Czernin J, Allen-Auerbach M, Schelbert HR. Improvements in cancer staging with PET/CT: literature-based evidence as of September 2006. J Nucl Med. 2007;48 Suppl 1:78S-88.

16. Chen W, Jian W, Li HT, Li C, Zhang YK, Xie B, et al. Whole-body diffusion-weighted imaging vs. FDG-PET for the detection of nonsmall-cell lung cancer. How do they measure up? Magn Reson Imaging. 2010;28:613-20.

17. Van Goethem M, Tjalma W, Schelfout K, Verslegers I, Biltjes I, Parizel P. Magnetic resonance imaging in breast cancer. Eur J Surg Oncol. 2006;32:901-10.

18. Heusner TA, Kuemmel S, Koeninger A, Hamami ME, Hahn S, Quinsten A, et al. Diagnostic value of diffusion-weighted magnetic resonance imaging (DWI) compared to FDG PET/CT for whole-body breast cancer staging. Eur J Nucl Med Mol Imaging. 2010;37:1077-86.

19. Heusner TA, Kuemmel S, Umutlu L, Koeninger A, Freudenberg LS, Hauth EA, et al. Breast cancer staging in a single session: wholebody PET/CT mammography. J Nucl Med. 2008;49:1215-22.

20. Hofmann M, Pichler B, Schölkopf and Beyer T. Towards quantitative PET/MRI: a review of MR-based attenuation correction techniques. Eur J Nucl Med Mol Ima. 2009;36 Suppl 1: S93104.

21. Conti M. Focus on time-of-flight PET: the benefits of improved time resolution. Eur J Nucl Med Mol Imaging. 2011. doi:10.1007/ s00259-010-1711-y. erratum doi:10.1007/s00259-011-1763-7.

22. Pichler BJ, Wehrl HF, Judenhofer MS. Latest advances in molecular imaging instrumentation. J Nucl Med. 2008;49 Suppl $2: 5 \mathrm{~S}-23$. 